\title{
Dynamic Connectivity Patterns in Conscious and Unconscious Brain
}

\author{
Yuncong Ma, ${ }^{1, *}$ Christina Hamilton,, ${ }^{2, *}$ and Nanyin Zhang ${ }^{1,2}$
}

\begin{abstract}
Brain functional connectivity undergoes dynamic changes from the awake to unconscious states. However, how the dynamics of functional connectivity patterns are linked to consciousness at the behavioral level remains elusive. In this study, we acquired resting-state functional magnetic resonance imaging data during wakefulness and graded levels of consciousness in rats. Data were analyzed using a dynamic approach combining the sliding window method and $k$-means clustering. Our results demonstrate that whole-brain networks contained several quasistable patterns that dynamically recurred from the awake state into anesthetized states. Remarkably, two brain connectivity states with distinct spatial similarity to the structure of anatomical connectivity were strongly biased toward high and low consciousness levels, respectively. These results provide compelling neuroimaging evidence linking the dynamics of whole-brain functional connectivity patterns and states of consciousness at the behavioral level.
\end{abstract}

Keywords: anesthetic-induced unconsciousness; dynamic functional connectivity; rat; resting-state networks

\section{Introduction}

B RAIN FUNCTION UNDERGOES dramatic changes from conscious to anesthetic-induced unconscious (AIU) states. Changes in brain function during AIU are intrinsically dynamic, considering that consciousness is known to be not an all-or-none, but a graded phenomenon (Laureys et al., 2007). However, what these dynamics are, and how dynamical changes in brain function are linked to consciousness at the behavioral level, remain unknown.

In recent years, accumulating evidence suggests that AIU is tightly associated with functional connectivity (FC) changes in brain networks (Lee et al., 2013; Liang et al., 2013; Mashour and Alkire, 2013; White and Alkire, 2003). These functional brain networks have been uncovered by neuroimaging techniques, especially resting-state functional magnetic resonance imaging (rsfMRI) (Boveroux et al., 2010), which measures resting-state FC (RSFC) based on temporal synchronizations of blood oxygen level-dependent (BOLD) signals from different brain regions (Biswal et al., 1995; Fox and Raichle, 2007). Although rsfMRI has been extensively used to study brain function during AIU, the conventional correlation analysis that rsfMRI uses implicitly assumes that RSFC is stationary during data acquisition.
Therefore, in essence, such analysis only provides averaged RSFC over the recording period, whereas the information of how RSFC temporally varies is overlooked. Given this nature, the most majority of previous rsfMRI studies focused on identifying steady-state connectivity changes between two or three anesthetic depths (Liang et al., 2012b; Vincent et al., 2007).

Despite the tremendous progress the steady-state rsfMRI paradigm has made, it may not be optimal to reveal the dynamic changes of brain network connectivity when the consciousness level is modulated. This limitation has highlighted a critical knowledge gap as the dynamics of wholebrain network connectivity at and in transition to different levels of consciousness will help decipher network reconfigurations when consciousness is perturbed. Therefore, this information can provide critical insight into understanding the systems-level neural mechanisms of AIU.

Recent progress in rsfMRI methodology demonstrates that dynamic information of brain connectivity can be reliably detected in humans and animals with novel analysis methods, such as the sliding window and coactivation pattern approaches (Allen et al., 2014; Chang and Glover, 2010; Hutchison et al., 2013a, b; Keilholz et al., 2013; Liang et al., 2015a; Liu and Duyn, 2013; Thompson et al., 2013, 2014). These methods

\footnotetext{
${ }^{1}$ Department of Biomedical Engineering, Pennsylvania State University, University Park, Pennsylvania.

${ }^{2}$ The Huck Institutes of Life Sciences, Pennsylvania State University, University Park, Pennsylvania.

*These authors contributed equally to this work.
} 
have made it feasible to investigate the brain network dynamics at different states of consciousness (Barttfeld et al., 2015; Liang et al., 2015a). Indeed, with these approaches, it has been shown that the dynamic properties of brain network connectivity may constitute key characteristics of consciousness (Barttfeld et al., 2015; Breshears et al., 2010; Hudetz et al., 2015).

To comprehensively elucidate the dynamics of brain connectivity patterns in different states of consciousness, in this study, we acquired rsfMRI data in rats during wakefulness and five graded levels of consciousness induced by increasing concentrations of isoflurane. The animal's state of consciousness was assessed using the loss of righting reflex (LORR) test. rsfMRI data were analyzed using the sliding window method combined with $k$-means clustering (Allen et al., 2014). We identified two specific brain network patterns that were strongly associated with high and low consciousness states, respectively. Interestingly, we also found a brain connectivity state that was dominant at the critical transitional point between the conscious and unconscious states. Based on these findings, we further analyzed the temporal sequences of separate connectivity patterns between conscious and unconscious states.

\section{Materials and Methods}

\section{Animal preparation}

All procedures were conducted in accordance with approved protocols from the Institutional Animal Care and Use Committee of the Pennsylvania State University. Twenty-five adult male Long-Evans rats $(300-500 \mathrm{~g})$ were housed and maintained on a 12-h light-12-h dark schedule, and provided access to food and water ad libitum throughout the duration of the study. To minimize stress and motion during imaging at the awake state, animals were acclimated to the scanning environment for 7 days (described in Liang et al., 2011, 2012a, 2013, 2014, 2015b; Ma et al., 2016; Zhang et al., 2010). To do this, rats were restrained and placed in a mock MRI scanner, where prerecorded MRI sounds were played. The exposure time in the mock scanner started from $15 \mathrm{~min}$ on day 1 , and was increased by $15 \mathrm{~min}$ each day up to $60 \mathrm{~min}$ (days $4,5,6$, and 7). This setup mimicked the scanning conditions inside the magnet.

\section{Behavioral assessment}

The animal's consciousness state was assessed using the behavioral test of LORR in a subgroup of animals that were imaged $(n=16)$. A strong correlation has been shown between the concentration of various anesthetic agents necessary for rodents to lose voluntary movement and loss of consciousness in humans (Franks, 2008). In the LORR test, each rat began in the awake state in the restrainer and was exposed to increasing doses of isoflurane through a nose cone. The LORR for a single dose was measured exactly as it was delivered to the animal inside the scanner (Fig. 1A). Each rsfMRI scan was 10-min long with a 5-min interscan transition time. Thus, for instance, LORR at $1.5 \%$ isoflurane was measured by sequentially delivering $0.5 \%$ and $1 \%$ isoflurane to the animal for $15 \mathrm{~min}$, respectively, followed by $1.5 \%$ isoflurane for $5 \mathrm{~min}$. With the nose cone in place, the rat was then removed from the restrainer and placed in a supine position while the time it took to correct its position was measured. The animal was deemed completely unconscious if it did not correct its position within $60 \mathrm{sec}$. This procedure mimicked anesthesia administration during the rsfMRI scanning process, and thus allowed us to verify the animal's consciousness state during each rsfMRI scan.

\section{MRI experiment}

Rats were briefly anesthetized with isoflurane while they were placed in a head restrainer with a built-in birdcage coil. Isoflurane was discontinued after the animal was set up, but the nose cone remained in front of the animal's nose for the duration of the whole MRI experiment. Imaging began $30 \mathrm{~min}$ after rats were placed in the scanner while animals were fully awake. Image acquisition was performed on a 7T scanner interfaced with a Bruker console (Billerica, MA). First, a T1weighted structural image was acquired with the following parameters: repetition time $(\mathrm{TR})=2125 \mathrm{~ms}$, echo time $(\mathrm{TE})=50$ $\mathrm{ms}$, matrix size $=256 \times 256$, field of view $(\mathrm{FOV})=3.2 \times 3.2 \mathrm{~cm}$, slice number $=20$, and slice thickness $=1 \mathrm{~mm}$. Subsequently, six rsfMRI scans were acquired: one as animals were fully
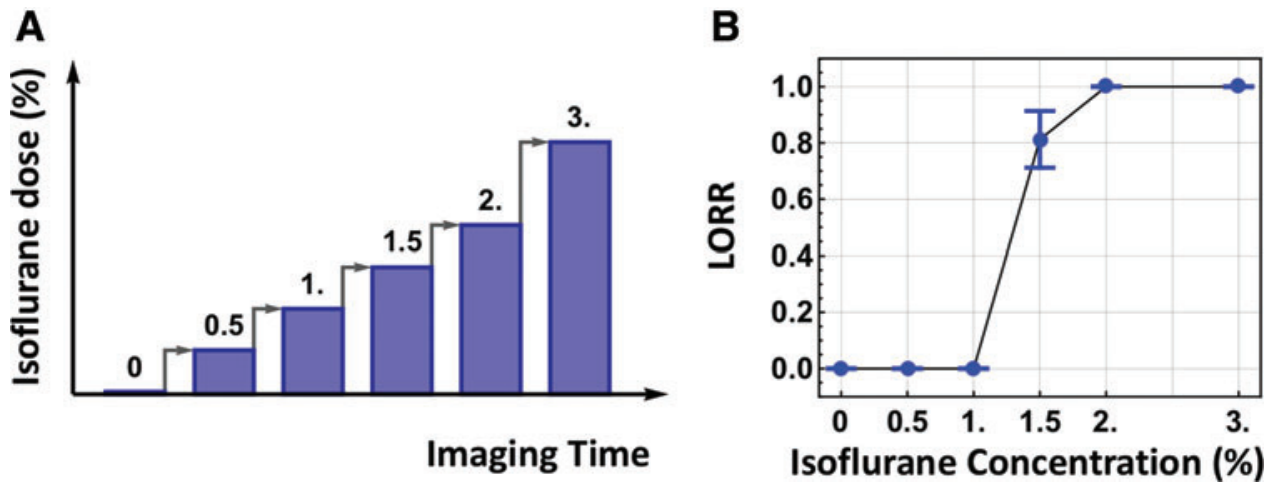

FIG. 1. rsfMRI paradigm and behavioral test. (A) The imaging paradigm consisted of six sequential rsfMRI scans with an increasing dose of isoflurane. Each 10-min scan was preceded by a 5-min interscan interval. (B) The fraction of the number of animals that lost righting reflex at each dose. Bars: S.E.M. rsfMRI, resting-state functional magnetic resonance imaging; S.E.M., standard error of the mean. Color images available online at www.liebertpub.com/brain 
awake and five at increasing isoflurane concentrations delivered at $0.5 \%, 1.0 \%, 1.5 \%, 2.0 \%$, and $3.0 \%$, respectively, with a 5-min transition time between scans (Fig. 1A). Isoflurane was delivered through the nose cone. rsfMRI data were acquired using a single-shot gradient echo echo-planar imaging pulse sequence with $\mathrm{TR}=1000 \mathrm{~ms}, \mathrm{TE}=13.8 \mathrm{~ms}$, flip angle $=80^{\circ}$, matrix size $=64 \times 64$, FOV $=3.2 \times 3.2 \mathrm{~cm}^{2}$, slice number $=20,1-\mathrm{mm}$-thick slices (in-plane resolution $=$ $\left.0.5 \times 0.5 \mathrm{~mm}^{2}\right)$. Six hundred rsfMRI volumes $(10 \mathrm{~min})$ were acquired for each scan (Fig. 1).

All imaging and behavioral data were also used in another study (Hamilton et al., 2016) and reanalyzed for the purpose of this article. The focus of Hamilton's article is on the global-scale information exchange loss during graded anesthesia, and there is no overlap in terms of analysis and findings between the two studies.

\section{Data analysis}

Image preprocessing. The first 10 volumes of each rsfMRI scan were removed to allow magnetization to reach a steady state. rsfMRI data were then manually coregistered to a standard rat atlas using the Medical Image Visualization and Analysis software (MIVA, http://ccni.wpi.edu), motion corrected using SPM 12 (University College London, United Kingdom), and then spatially (Gaussian kernel, sigma $=0.5 \mathrm{~mm}$ ) as well as temporally smoothed $(0.01-0.1 \mathrm{~Hz})$. In addition, signals from white matter and ventricles, six motion parameters estimated from motion correction (translation and rotation), as well as their derivatives were regressed out (Power et al., 2015).

Brain parcellation. The whole brain was parcellated into 134 unilateral regions of interest (ROIs) based on the anatomic definition of brain regions in the Swanson Atlas (Swanson, 2004). One hundred ten of these ROIs were further grouped into nine brain systems, including sensorymotor, polymodal association, retrohippocampal regions, hippocampus, amygdala complex, striatum, pallidum, thalamus, and hypothalamus (Liang et al., 2012b) (Fig. 2A). The remaining 24 ROIs were noted as "others." The ROI time course was extracted by regionally averaging rsfMRI time series from all voxels within the ROI.
A

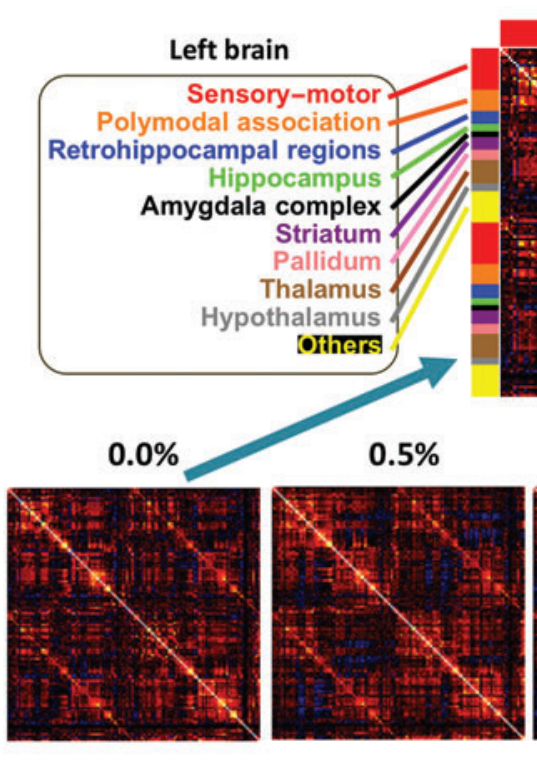

C

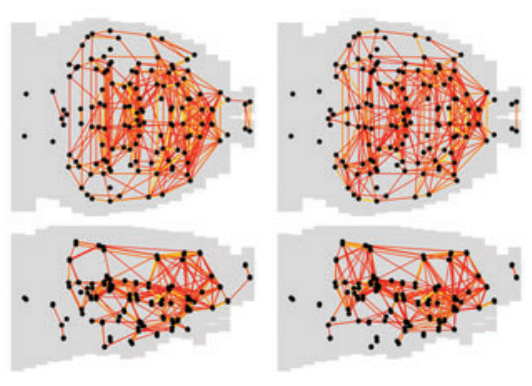

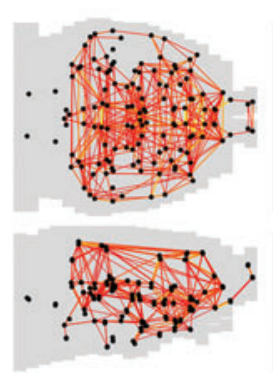
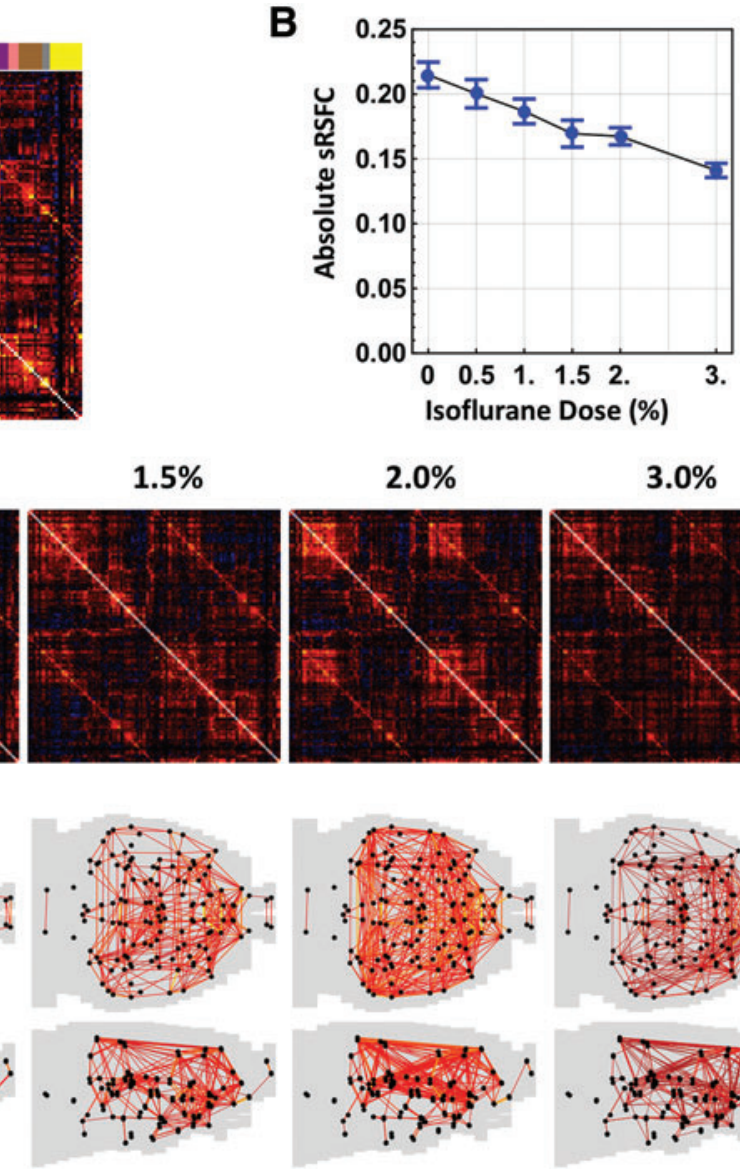

$1.5 \%$
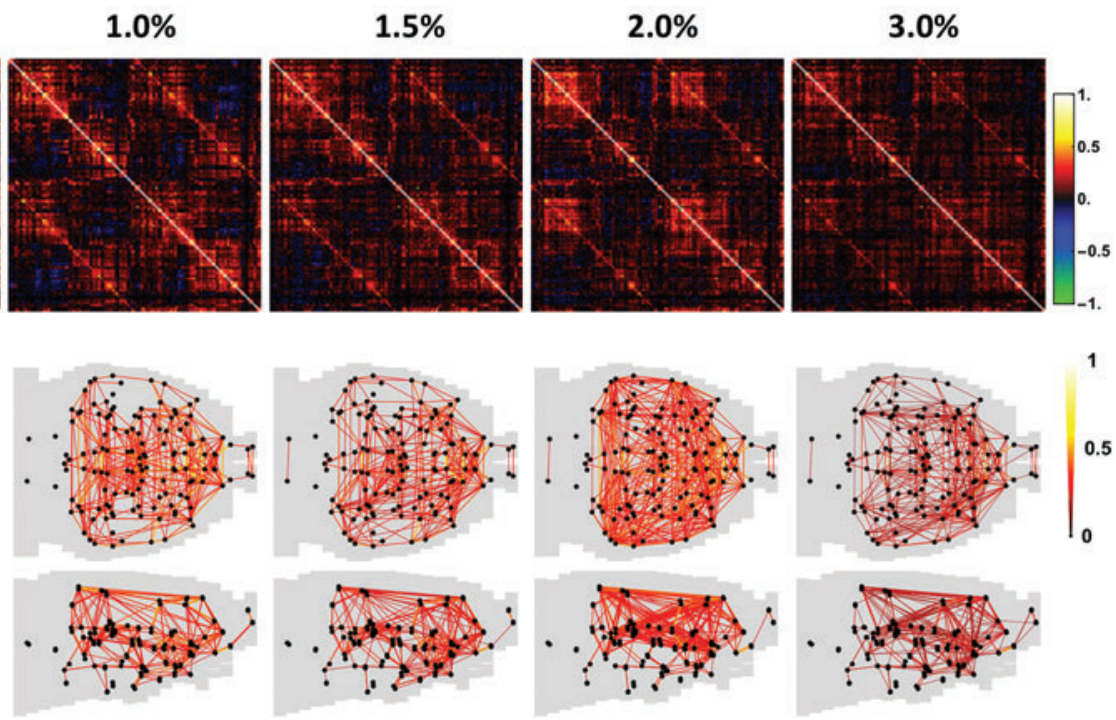

FIG. 2. sRSFC patterns. (A) sRSFC matrices at the awake state $(0.0 \%)$ and five anesthetic depths induced by increasing concentrations of isoflurane $(0.5 \%, 1.0 \%, 1.5 \%, 2.0 \%$, and $3.0 \%)$. ROIs were grouped by the hemisphere and brain system (first half: left hemisphere, second half: right hemisphere). Red: sensory-motor system, orange: polymodal-association system, blue: retrohippocampal regions, green: hippocampus, black: amygdala complex, purple: striatum, pink: pallidum, brown: thalamus, gray: hypothalamus, yellow: all other ROIs. (B) Average absolute sRSFC strength during wakefulness and five isoflurane concentrations, bars: S.E.M. (C) Axial and sagittal views of brain connectivity patterns for all conditions. Dark dots indicate ROIs. For each dose, 446 strongest connections (i.e., 0.05 graph density) were displayed. Color images available online at www.liebertpub.com/brain 
Estimating anatomical connectivity based on axonal projection density. We used the Allen Mouse Brain Connectivity Atlas to generate the structure of anatomical connectivity between ROIs based on axonal projection density. The Allen Brain Atlas was used due to its public availability and the high anatomical and functional similarity between rat and mouse brains (Oh et al., 2014). Considering that the Allen mouse's anatomical map contains finer brain structures that cannot be differentiated by our fMRI's spatial resolution, we first combined all small substructures in the Allen Brain Atlas that belonged to each ROI defined in the rat brain. Second, the average axonal projection density from ROI A to ROI B was assessed by taking the weighted average of the projection densities across all substructures in A to all substructures in B, with the size of each substructure in B as the weight. Mathematically, the average projection density from A to B was defined as:

$$
P D_{A, B}=\left(\sum_{i=1}^{N_{A}} \sum_{j=1}^{N_{B}} P D_{A, i, B, j} \text { Area }_{B, j}\right) / \sum_{j=1}^{N_{B}} \text { Area }_{B, j},
$$

where $P D_{A, i, B, j}$ is the projection density from the $i$-th substructure in ROI A to the $j$-th substructure in ROI B; Area $_{B, j}$ is the size of the $j$-th substructure in ROI B; $N_{A}$ and $N_{B}$ are the number of substructures in ROI A and B, respectively. Finally, the structural connectivity strength between ROI A and $\mathrm{B}$ was calculated as the mean of $P D_{A, B}$ and $P D_{B, A}$.

Deriving brain FC. We first calculated the brain FC patterns under the stationarity assumption. The ROI-wise RSFC matrices (referred to as sRSFC matrices hereafter) were obtained by calculating the Pearson correlation coefficients between each pair of ROI time courses at the awake and five anesthetic depths. These FC matrices were organized by the brain systems defined above (Fig. 2).

Dynamic brain connectivity patterns in different anesthetized states were analyzed using the sliding window method ( window length $=60 \mathrm{sec}$, step size $=5 \mathrm{sec}, \mathrm{TR}=1 \mathrm{sec}$ ). A 60 -sec window size was selected based on the report that window sizes around $30-120 \mathrm{sec}$ produce robust RSFC (Hutchison et al., 2013a; Jones et al., 2012; Shirer et al., 2012). For each rsfMRI scan, 61 windows were extracted for each dose. The RSFC matrix of each window was calculated in the same manner as sRSFC matrices. RSFC matrices for all windows in the awake state and five anesthetic depths from all animals were then pooled together and clustered into five temporarily alternating, but spatially repeatable wholebrain connectivity patterns (referred to as dRSFC hereafter) using $k$-means clustering (Manhattan distance). These dRSFC patterns were ranked according to their spatial similarity (measured by Manhattan distance) to the structural connectivity map obtained as mentioned above. To facilitate the comparison between dRSFC and structural connectivity, an arctangent function was applied to normalize the dRSFC strength and anatomical projection intensity, respectively. For each dRSFC pattern, the occurrence rate was measured for each consciousness state.

To explore the temporal transitions between dRSFC patterns, the state sequence of each scan, which represents the sequence of rsfMRI windows with the identity of their corresponding dRSFC patterns, was obtained from the clustering results. The number of transitions from one state to another state, which was apart by one window length (i.e., $60 \mathrm{sec}$ ), was counted. The 60 -sec gap ensures the temporal independence of the two brain connectivity states in transition counts. The state transition probability from one state to another was then calculated after excluding all self-transitions.

Statistics. Isoflurane dose effects were statistically tested using chi-square test or one-way analysis of variance (ANOVA). The relationship between dynamic RSFC patterns and consciousness levels were tested using two-way ANOVA. The comparison between high and low consciousness levels was conducted using two-sample $t$-tests. The statistical significance was set at $p<0.05$.

\section{Results}

In the present study, we examined the anesthetic-induced dynamic changes in the whole-brain network connectivity as animals transitioned from the awake state into an unconscious state. rsfMRI data during wakefulness and graded levels of consciousness produced by increasing concentrations of isoflurane $(0.5 \%, 1.0 \%, 1.5 \%, 2.0 \%$, and $3.0 \%)$ were collected in rats.

\section{Assessing the consciousness level at different anesthetized states}

To understand the dynamics of network connectivity at different consciousness levels, we determined the animal's conscious state at each isoflurane dose delivered. The animal's consciousness level was measured using LORR outside of the scanner in a manner that directly mimicked the way the dose was delivered during rsfMRI scanning ( $n=16$, Fig. 1A, also see Methods section). Our data showed that no rats lost righting reflex when $1 \%$ or $0.5 \%$ isoflurane was delivered through a nose cone, whereas $81 \%$ of animals (13 out of 16) were unable to correct from the supine to prone position when $1.5 \%$ isoflurane was delivered. All animals lost righting reflex at higher delivered doses $(2 \%$ and $3 \%$, Fig. 1B). Therefore, loss of consciousness was induced when $1.5 \%$ isoflurane was delivered through a nose cone (chi-square test, $\chi^{2}=22.82, p<2 \times 10^{-6}$ ).

\section{Stationary RSFC strength was reduced during AIU, although the spatial pattern of whole-brain network remained similar}

We first examined the steady-state brain FC under the stationarity assumption. The whole brain was parcellated into 134 unilateral ROIs (Swanson, 2004). The ROI-wise RSFC matrices (referred to as sRSFC matrices) were obtained by calculating the Pearson correlation coefficients between pairs of ROI time courses of each rsfMRI scan at the awake state and five anesthetic depths. Consistent with previous studies (Liang et al., 2012b; Liu et al., 2011; Lu et al., 2007; Peltier et al., 2005), sRSFC decreased at increasing anesthetic dose (Fig. 2A, B). The absolute strength of sRSFC averaged across all connections significantly decreased in a monotonic manner as the isoflurane concentration increased (Fig. 2B, one-way ANOVA: $F=8.48, p<10^{-6}$ ). However, the sRSFC spatial patterns remained similar across different consciousness states (Fig. 2C), reflected by a high spatial correlation coefficient between sRSFC matrices averaged across all conditions [mean $( \pm \mathrm{SD})=0.75( \pm 0.07)]$. 


\section{Whole-brain connectivity patterns contained several quasi-stable states that dynamically alternated from the awake state into anesthetized states}

As largely unaltered sRSFC patterns did not seem to be able to explain dramatic behavioral change from wakefulness to unconsciousness, we further examined dynamic whole-brain connectivity patterns across all conditions. rsfMRI data at each condition were segmented using a sliding window (window length $=60 \mathrm{sec}$, step size $=5 \mathrm{sec}$, $\mathrm{TR}=1 \mathrm{sec})$. For each window, RSFC between each pair of ROIs was calculated using the Pearson correlation coefficient between the ROI time courses. This step yielded the RSFC matrix (i.e., a whole-brain connectivity pattern) for the window. RSFC matrices for all windows at the awake state and five anesthetic depths across all animals were then pooled together and clustered into five whole-brain connectivity patterns using $k$-means clustering (Manhattan distance, $k=5$ ). These five temporarily alternating but spatially repeatable connectivity patterns were then ranked according to their spatial similarity (Manhattan distance) to the structural connectivity map generated based on axonal projection densities (Oh et al., 2014) (Fig. 3).

These dynamic connectivity patterns (referred to as dRSFC) were considerably different from the sRSFC matrices, suggesting that the brain network connectivity displayed dynamic changes that were not revealed by the conventional correlation analysis based on the stationarity assumption. In addition, all five dRSFC patterns recurred in all conditions. These results collectively indicate that whole-brain connectivity patterns contained several quasi-stable states that were spatially repeatable, but temporally alternating from the awake state into anesthetized states.
Occurrence of dRSFC patterns exhibited strong bias to different consciousness levels

We assessed the occurrence rate of all five dRSFC patterns at each conscious condition to understand the relationship of dRSFC patterns to different consciousness levels. Although all five dRSFC patterns shown in Figure 3 were recurring in all conditions, their occurrence rate exhibited strong bias toward different consciousness levels (two-way ANOVA: $F=17.7, \quad p<10^{-13}$ for the effect of dRSFC patterns; $F=9.46, p<10^{-25}$ for the interaction between dRSFC patterns and consciousness levels). Intriguingly, we found that dRSFC patterns 1 and 5 (Fig. 4A), which were the least and most similar to the structural connectivity map, respectively, had reversed occurrence probability from the awake to unconscious states (Fig. 4C). dRSFC pattern 1 was more likely to occur when animals were at higher consciousness levels and the occurrence probability of this state decreased dramatically as the anesthetic depth increased, approaching zero at the isoflurane concentration of $3.0 \%$ (one-way ANOVA: $F=10.6, p=10^{-7}$ ). Conversely, dRSFC pattern 5 had a higher occurrence rate when animals became less conscious, and was the predominant connectivity pattern at the deepest anesthetic level (one-way ANOVA: $F=12.2$, $p=10^{-9}$ ).

To further elucidate this bias in occurrence rate, all six conditions (one awake and five anesthetic conditions) were grouped into two consciousness levels based on the LORR assessment (Fig. 1B), with conditions of isoflurane $0 \%$ (i.e., wakefulness), $0.5 \%$, and $1 \%$ assigned to the high consciousness group, and isoflurane $1.5 \%, 2 \%$, and $3 \%$ assigned to the low consciousness group. Figure 4B illustrates a statistically significant difference between the occurrence rate of dRSFC patterns 1 and 5 during the two consciousness levels

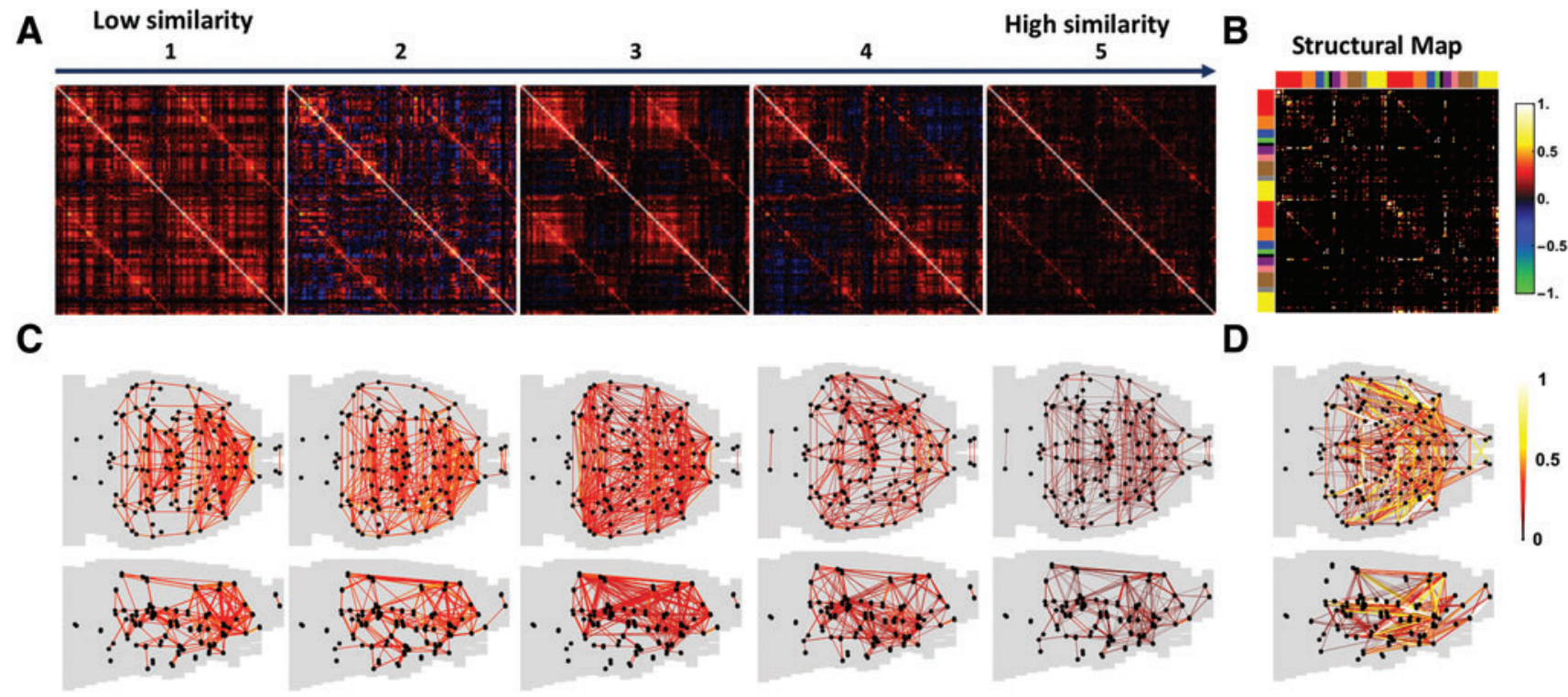

FIG. 3. Five dRSFC patterns derived from $k$-means clustering across all conditions. (A) Five recurring dRSFC patterns ranked by their spatial similarity to (B) structural connectivity. One hundred thirty-four ROIs were grouped by the hemisphere and brain system in the same way for Figure 2. (C) Axial and sagittal views of all dRSFC patterns. (D) Axial and sagittal views of anatomical connectivity. In (C) and (D), 446 strongest connections (graph density $=0.05)$ in each brain network are displayed. ROIs, regions of interest. Color images available online at www.liebertpub.com/brain 


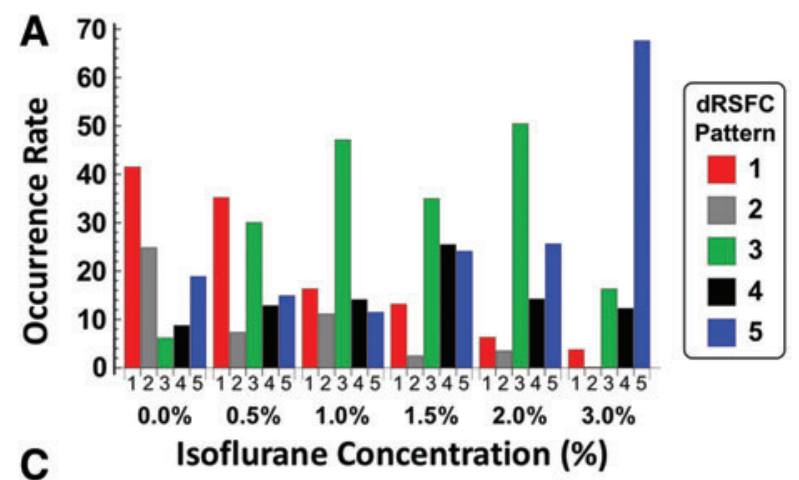

B

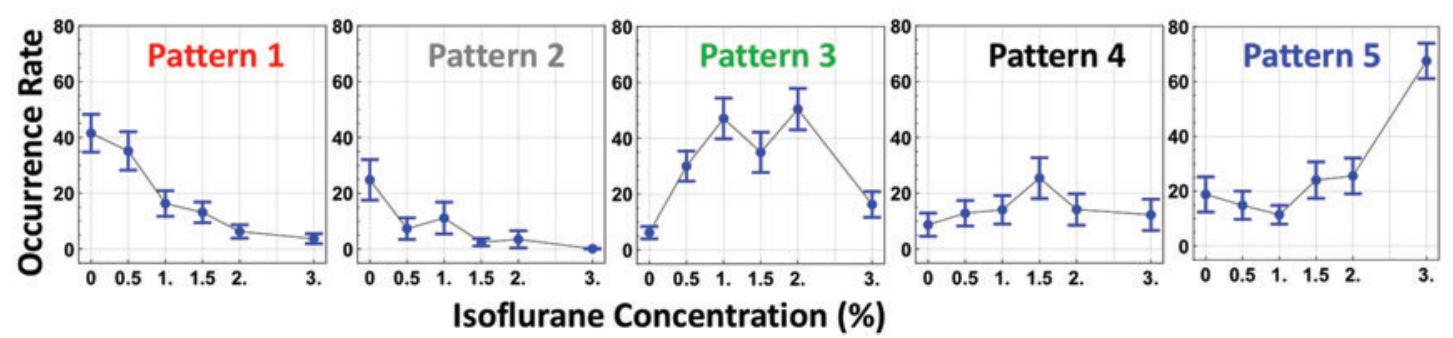

FIG. 4. (A) Occurrence rate for all five dRSFC patterns at each anesthetic dose. $0.0 \%$ indicates the awake state. (B) Occurrence rates of patterns 1 and 5 at two consciousness levels grouped based on the behavioral test result. Isoflurane concentrations $0.0 \%, 0.5 \%$, and $1.0 \%$ were assigned to the group of high consciousness level, and $1.5 \%, 2 \%$, and $3 \%$ were assigned to the group of low consciousness level. The occurrence probability between the two dRSFC patterns was statistically different for both consciousness levels $\left(p<0.001\right.$ for high consciousness level and $p<10^{-8}$ for the low consciousness level). (C) Occurrence rate as a function of anesthetic depth for each dRSFC pattern, bars: S.E.M. ***p<0.001. Color images available online at www.liebertpub.com/brain

(two-sample $t$-test: $p<10^{-3}$ for the high consciousness level, $p<10^{-8}$ for the low consciousness level).

\section{dRSFC pattern 3 represents a transition state between the conscious and unconscious states}

Interestingly, in addition to two brain connectivity states (dRSFC patterns 1 and 5) that were respectively characteristic to high and low consciousness levels, we observed a third connectivity state (dRSFC pattern 3 ) whose occurrence rate had a bell-shaped distribution centered at $1.5 \%$ isoflurane (one-way ANOVA: $F=8.28, p<10^{-6}$ ), the dose delivered when rats transitioned from the conscious state into the unconscious state (Fig. 4C). This result suggests that this dRSFC pattern may represent a critical transitional state that occurs when the consciousness state undergoes dramatic changes at the behavioral level (Fig. 1).

To understand the possible transition role of dRSFC pattern 3 in switching between conscious and unconscious states, the temporal sequence of brain connectivity states for all rsfMRI windows were extracted. Each brain connectivity state was represented by the dRSFC pattern that the rsfMRI window belonged to. We calculated the state transition matrix to measure the transition probability between each two states (Fig. 5A, self-transitions were excluded). The transition probability indicates that state 1 was more likely to transition to state 3 ( $42 \%$ chance) than any other states, and so was state 5 (60\% chance). Relative to random transitions permuted between all states while maintaining the transition probability, the preference of these transition pathways was statistically significant (state 1 to state 3 : chi- square test: $\chi^{2}=41.7, p<10^{-7}$; state 5 to state 3 : chi-square test: $\left.\chi^{2}=77.6, p<10^{-15}\right)$. In addition, state 3 was most likely to transition to states 1 or 5 (78\% chance combined, chi-square test: $\left.\chi^{2}=66.8, p<10^{-12}\right)$. Moreover, transitions between states 1 and 5 were predominantly through two pathways: (1) transition through state 3 , or (2) direct transition (combined $95.8 \%$ chance, Fig. 5B). Taken together, these results indicate that in addition to direct transitions between dRSFC patterns 1 and 5, dRSFC pattern 3 might represent an intermediate connectivity state between the conscious and unconscious states.

\begin{tabular}{c|ccccc} 
A & \multicolumn{1}{c}{ Transition Matrix } \\
TP $(\%)$ & 1 & 2 & 3 & 4 & 5 \\
\hline 1 & 0 & 13 & 42 & 16 & 29 \\
2 & 64 & 0 & 15 & 10 & 11 \\
3 & 31 & 9 & 0 & 13 & 47 \\
4 & 29 & 3 & 41 & 0 & 28 \\
5 & 24 & 4 & 60 & 12 & 0
\end{tabular}

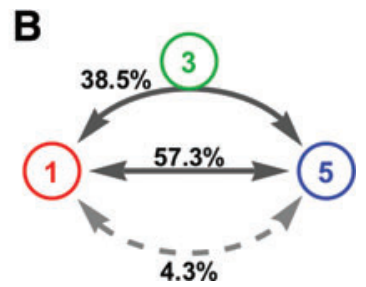

FIG. 5. State transitions. (A) Transition matrix between five brain connectivity states. Self-transitions were not counted. (B) Occurrence probability of different transition pathways between brain connectivity states 1 and 5 . The upper line indicates that the transition path was through brain connectivity state 3 . The lower line indicates the transition path was through either brain connectivity states 2 or 4 , or both. The middle line suggests a direction transition between brain connectivity states 1 and 5 . Color images available online at www.liebertpub.com/brain 
A

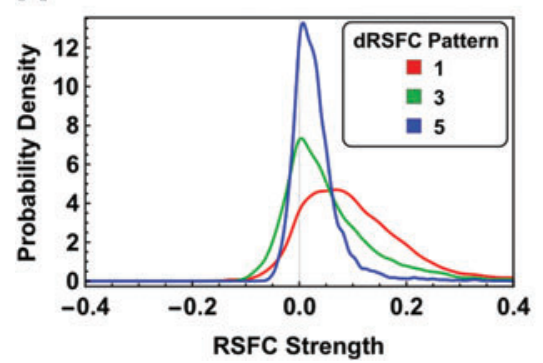

B

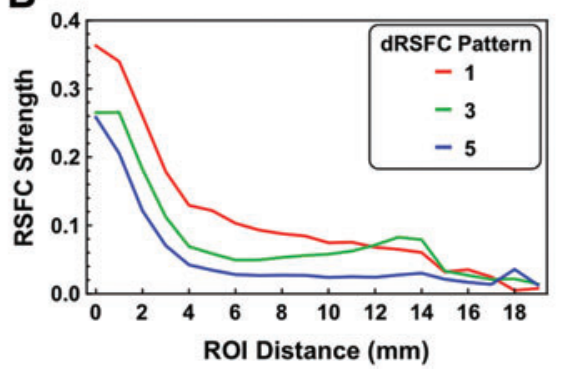

C

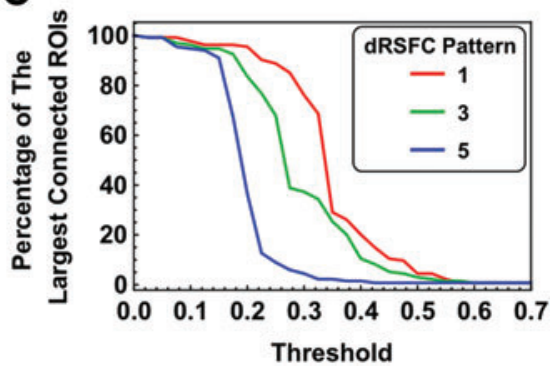

FIG. 6. Connectivity characteristics of dRSFC networks. (A) Distribution of RSFC strength for dRSFC networks 1, 3, and 5. (B) Average absolute connectivity strength as a function of physical distance of all connections. (C) The maximal percentage of connected ROIs at different RSFC thresholds for all dRSFC networks. Color images available online at www.liebertpub.com/brain

dRSFC patterns displayed distinct organizational architecture

To further understand the organizational architecture of dRSFC patterns of interest (patterns 1,3, and 5), we estimated their connectivity strength distribution, the relation- ship between strength and connection distance, robustness, as well as the topological organization (Figs. 6 and 7). Among all three patterns, pattern 1 displayed the most heterogeneous distribution in connectivity strength (Fig. 6A). This result is consistent with the notion that the awake state is associated with a richer functional repertoire (Barttfeld et al.,
A dRSFC

1

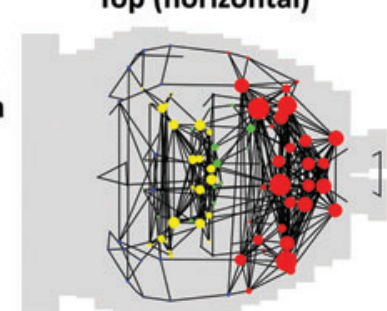

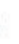

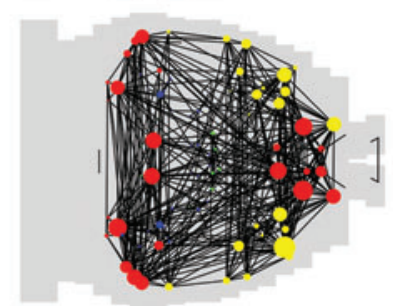

Right (sagittal)
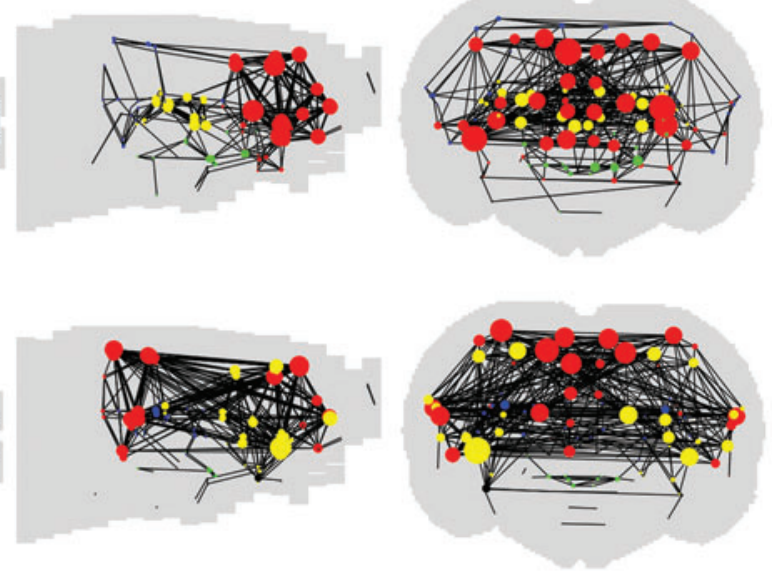

Front (coronal)

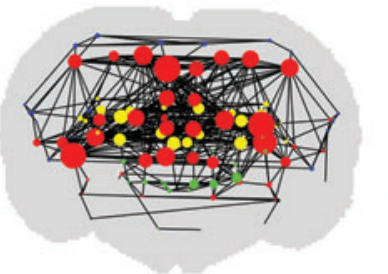

Community
1
2
3
4
4
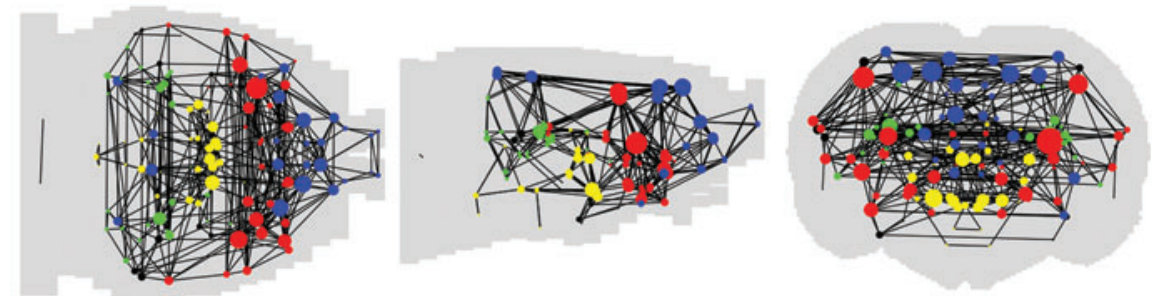

B
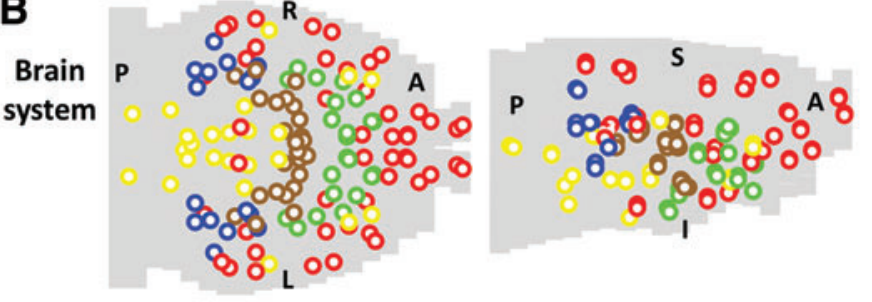

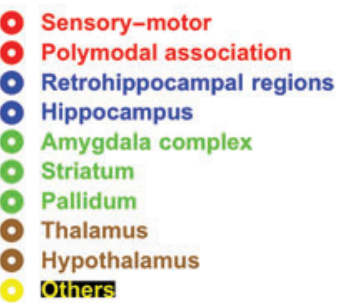

- Sensory-motor

- Polymodal association

- Retrohippocampal regions

Hypothalamus orthers

FIG. 7. Community structures of dRSFC networks 1,3 , and 5 (at the graph density $=0.05$ ). (A) Communities were color labeled. The sphere size represents the degree of the ROI. (B) The nine brain anatomical systems were displayed in four colors for the reference purpose. A, anterior; L, left; P, posterior; R, right; S, superior; I, inferior. Color images available online at www.liebertpub.com/brain 
2015; Hudetz et al., 2015). The averaged absolute connectivity strength as a function of physical distance between ROIs was shown in Figure 6B for all three dRSFC patterns. In general, all three connectivity patterns showed a trend of decreasing connectivity strength for longer-distance connections. However, state 3 displayed a "rebound" of RSFC strength for certain long-distance connections.

To assess the robustness of dRSFC networks, we calculated the percentage of ROIs of the largest connected component relative to the total ROI number at different RSFC strength thresholds (Fig. 6C). In this analysis, two ROIs were deemed disconnected if the RSFC strength between them was below the threshold. This analysis can highlight the impact of losing weak connections on the network. For all three dRSFC networks, the whole-brain network began to fall into unconnected components when the threshold was elevated to a certain level. Among these three dRSFC networks, the robustness decreased in the order of network 1,3 , and 5 , reflected by a gradually smaller threshold needed to fragment the network.

To examine the topological organization of dRSFC networks, we calculated the community structure of the three dRSFC networks under the same graph density (0.05). Pattern 1 primarily consisted of two communities (Fig. 7A), with one community (red) containing the sensory-motor, polymodal association, and striatum systems, and the other community (yellow) containing the thalamus and hippocampus. dRSFC network 3 was characterized by three communities. The first community (red) contained brain regions in the sensory-motor, polymodal association, and retrohippocampal systems. The second community (yellow) included the sensory-motor, polymodal association and striatum systems. The third community (blue) contained part of thalamus and hippocampus. State 5 had four representative communities. Community 1 (red) included the sensory-motor, polymodal association, striatum, and pallidum. Community 2 (blue) contained the sensory-motor and polymodal systems. Community 3 (yellow) included hypothalamus and a small portion of thalamus. Community 4 (green) included thalamus, hippocampus, and retrohippocampal regions.

\section{Characteristic dRSFC patterns were robust regardless of the clustering number}

To examine the possible influences of the clustering number selected during $k$-means clustering $(k=5)$, we also obtained results using different clustering numbers (see Supplementary Fig. S1; [Supplementary Data are available online at www .liebertpub.com/brain] for the case of $k=3$, and Supplementary Fig. S2 for the case of $k=7$ ). In both cases, highly consistent dRSFC spatial patterns and occurrence preference in relation to consciousness states were revealed. At $k=3$, three dRSFC patterns, respectively, coincided with patterns 1,3 , and 5 in the case of $k=5$ in terms of spatial patterns (the spatial correlations between the corresponding dRSFC patterns at $k=3$ and $k=5$ were $0.957,0.996$, and 0.917 , respectively) and occurrence probability at different consciousness levels. At $k=7$, the same three patterns were also identified (patterns 2, 6 , and 7 , the spatial correlations with dRSFC pattern 1,3 , and 5 at $k=5$ were $0.948,0.974$, and 0.981 , respectively). Taken together, these results suggest that characteristic dRSFC patterns identified were robust regardless of the clustering number.

\section{Discussion}

The brain is an intrinsically dynamic system characterized by a variety of spatiotemporal connectivity patterns (Hutchison et al., 2013a). These FC patterns can provide important information for differentiating healthy and clinical populations (Anand et al., 2005; Carter et al., 2012; Greicius et al., 2004, 2007; Hunter et al., 2012; Kennedy et al., 2006; Lowe et al., 2002; Lustig et al., 2003; Mayer et al., 2011; Tian et al., 2006; van Meer et al., 2012; Whitfield-Gabrieli et al., 2009), or can act as a fingerprint capable of accurately identifying individual subjects from a large group (Finn et al., 2015). An intriguing question that remains elusive is how modulations of the level of consciousness are reflected in these dynamic connectivity patterns. In the present study, we investigated the dynamics of brain network connectivity that are associated with consciousness and unconsciousness at the behavioral level. We confirm the presence of several quasi-stable whole-brain connectivity states that dynamically alternated from the awake state into anesthetized states. In particular, two brain connectivity patterns were identified to be characteristic to conscious and unconscious states, respectively. In addition, they exhibited the opposite similarity to the structure of anatomical connectivity. Interestingly, we also found a brain connectivity state that plays an important role in transitions between conscious and unconscious states. Taken together, all these findings provide compelling neuroimaging evidence that contributes to the understanding of dynamic network changes across consciousness levels. These results suggest that specific brain connectivity patterns with distinct spatial similarities to anatomical connectivity might be characteristic to different states of consciousness.

\section{sRSFC strength decreased, but the connectivity pattern was maintained across conscious conditions}

First, we calculated the widely used sRSFC between ROIs at the awake state and five graded anesthetic depths induced by increasing concentrations of isoflurane (Fig. 2). Although the strength of sRSFC monotonically decreased with increasing isoflurane dose, we found that the spatial pattern of sRSFC generally remained similar across all conditions. This may be partially due to a global loss of information exchange under anesthesia, resulting in reduced RSFC, but maintaining a similar whole-brain connectivity pattern (Tononi, 2004). This finding is also consistent with a recent study reporting resembled connectivity patterns during wakefulness, anesthesia and sleep based on electrocorticographic $(\mathrm{ECoG})$ recordings from large cortical areas of macaque monkeys (Liu et al., 2014).

\section{Separate dRSFC patterns were characteristic to different states of consciousness}

Similar sRSFC patterns across different consciousness states can hardly explain the dramatic behavioral change from wakefulness to AIU (Keilholz, 2014). This is likely because AIU causes dynamic changes in brain function, whereas sRSFC data analysis can only provide averaged RSFC over the data acquisition period, losing all dynamic information. Therefore, to explore the possible link between RSFC and consciousness at the behavioral level, we used a dynamic rsfMRI approach combining the sliding window method and $k$-means clustering to reveal the brain's 
dRSFC patterns across all conscious conditions. Based on this analysis, we derived five brain connectivity patterns (Fig. 3) that were spatially repeatable and alternately exhibited during all states of consciousness, supporting the notion that brain network connectivity patterns contain dynamic but quasi-stable states (Allen et al., 2014; Liang et al., 2015a; Liu and Duyn, 2013; Majeed et al., 2011; Yuan et al., 2014).

Remarkably, even though all five brain connectivity patterns recurred in all states of consciousness, separate dRSFC patterns exhibited strong bias to different consciousness levels, reflected by their distinct occurrence preference at different isoflurane doses (Fig. 4). Specifically, we identified a dRSFC pattern (pattern 5) that displayed a high occurrence rate when the consciousness level is low, and became the dominant connectivity pattern at the highest isoflurane concentration. Conversely, another dRSFC pattern (pattern 1) frequently recurred when the consciousness level was high, and its occurrence rate dropped exponentially after the animal lost consciousness. Such results were observed regardless of the clustering number $(k)$. Taken together, our results suggest that dRSFC reveals connectivity patterns that are characteristic to the conscious and unconscious states, respectively, and thus can link dynamics of brain network connectivity to consciousness at the behavioral level (Figs. 3 and 4).

\section{dRSFC patterns characteristic to the conscious and unconscious states contained distinct similarities to anatomical connectivity}

Intriguingly, the dRSFC pattern that was strongly associated with the unconscious state was the most similar to the structural map among all five dRSFC patterns. This result is consistent with a recent finding reported by Barttfeld et al. (2015). In that study, it was suggested that FC observed during unconsciousness arises from "a semirandom circulation of spontaneous neural activity along fixed anatomical routes," and thus the brain's FC pattern during unconsciousness is dictated by the structure of anatomical connectivity. Additionally, the same study showed that the conscious state contained a richer repertoire of functional configurations, although there was no investigation of a detailed relationship between these configurations and the conscious state (Barttfeld et al., 2015). In the present study, we further identified a specific connectivity pattern (dRSFC pattern 1) that was strongly associated with the conscious state. More interestingly, we found that this state was the most dissimilar from the structural map and displayed the most heterogeneous distribution in connectivity strength, indicating that information communication between different brain regions in this connectivity state is far more complicated than the restriction of anatomical connections. Our data can also support the hypothesis that multisynaptic connectivity is preferentially disrupted at deeper anesthetic states, relative to monosynaptic connectivity. However, this hypothesis needs to be more rigorously tested in future studies.

The dominance of these two connectivity patterns in the conscious and unconscious states, respectively, was further confirmed by grouping all six conditions into two consciousness levels: high consciousness (wakefulness to $1.0 \%$ isoflurane) and low consciousness (1.5-3.0\% isoflurane). We showed that the occurrence rates of these two dRSFC patterns were significantly different between the two consciousness levels (Fig. 4B). Taken together, these results suggest that specific dRSFC patterns as well as their spatial similarities to structural connectivity might be characteristic to different states of consciousness.

\section{Brain connectivity state during the transition from wakefulness to unconsciousness}

Another interesting finding is that a brain connectivity state (dRSFC pattern 3) was dominant at the isoflurane dose regime, where animals were behaviorally determined to be losing consciousness, suggesting a possible relation to the transitional phase from wakefulness to unconsciousness (Fig. 4C). To confirm this transition role, we studied the state transitions, especially between dRSFC patterns 1 , 3 , and 5 (Fig. 5). First, transitions between these three states dominated all possible state transitions, even after considering the difference in occurrence rates. Second, transitions show particular preference between states 1 and 3 , as well as between states 3 and 5. Third, we found two typical transition pathways between states 1 and 5: (1) transition in a direct way and (2) transition through state 3 . Taken together, these results indicate that in addition to direction transitions between states 1 and 5 , brain connectivity state 3 likely plays a transitional role between conscious and unconscious states.

\section{Potential limitations}

Isoflurane concentrations received by animals were lower than concentrations delivered. Our data indicate that loss of consciousness was induced when $1.5 \%$ isoflurane was delivered through a nose cone. This dose was higher than typical isoflurane doses inducing LORR reported in the literature $(0.7-0.8 \%)$, in which animals were either intubated or were placed in an enclosed chamber (Hudetz et al., 2011; MacIver and Bland, 2014). This apparent contradiction most likely resulted from the difference in the setup for isoflurane delivery. In our study, we could not intubate the animals as they were also imaged at the awake state in the same scanning session and intubation will unavoidably cause pain. Therefore, a nose cone was used for isoflurane delivery. As a result, the actual isoflurane concentrations in the animal were considerably lower than the concentrations delivered as air can easily entrain through the nose cone and dilute the anesthetics. However, it has to be noted that the goal of the present study is not to investigate the connectivity pattern at a specific isoflurane concentration. Instead, our goal is to use graded isoflurane concentrations to obtain different consciousness levels and examine the dynamic FC patterns in these states. Therefore, what is more important is to determine the animal's conscious state during each rsfMRI scan. In our study, the animal's consciousness level during each rsfMRI scan was accurately measured using the LORR test outside of the scanner in the exact manner as the imaging experiment (Fig. 1). As a result, even though the isoflurane concentrations in the animal were lower than those delivered and not precisely determined, it should not affect any conclusions of the present study.

Potential impact of different physiological conditions between conscious states on imaging results. It is well 
known that anesthesia can considerably alter physiological conditions in animals. It is likely that different physiological conditions between the awake and anesthetized states can affect the connectivity pattern (Birn et al., 2008; Chang et al., 2009). However, our previous study suggests that influences of physiological states on RSFC in animals are minor due to several reasons (Liang et al., 2015a). First, it has been shown that the relative contributions from cardiac and respiratory noise to the rsfMRI results were $1 \%$ and $5 \%$, respectively, in the rat (Kalthoff et al., 2011), which are substantially smaller than the impact of physiological noise on human rsfMRI data (Birn et al., 2008; Chang et al., 2009). Second, in image preprocessing, we regressed out the signals from the white matter and ventricles to further minimize the influences of physiological noise (Chang and Glover, 2009). Third, difference in physiological conditions might induce steady-state changes in FC at each anesthetic dose. However, it is unlikely to cause systematic dynamic RSFC patterns found in the present study, particularly considering that all dRSFC patterns recurred in all anesthetic conditions. In fact, in our previous study (Liang et al., 2015a), we calculated the correlation between the BOLD signals from the white matter and ventricles and the time series of each individual voxels. The spatial pattern of this correlation departed far away from any dynamic connectivity patterns (awake or anesthetized) reported in that study, suggesting minimal influences from physiological noise on dynamic RSFC in rats. These results collectively indicate that difference in physiological conditions cannot account for any major effects of dRSFC patterns in the present study.

Impact of motion. Another potential pitfall is that dRSFC patterns could be attributed to the difference in the motion level at different anesthetic depths. Motion decreased as the anesthetic level increased, as shown in Supplementary Figure S3a. In addition, the motion level of dRSFC pattern 1 was higher than those of dRSFC patterns 3 and 5 (Fig. 1B), presumably because dRSFC pattern 1 was strongly biased by the awake state, while patterns 3 and 5 were more dominant in unconscious states. To rule out the possibility that characteristic dRSFC patterns identified in the present study were motion artifacts, we selected a subgroup of animals $(n=15)$, whose movement level was minimal in all rsfMRI scans. Three dRSFC patterns, with the same occurrence probability in relation to the consciousness level, were also identified in this subset of data (Supplementary Fig. S4, Patterns 3, 4, and 5, respectively, correspond to Patterns 1,3 , and 5 in the full dataset. Patterns 1 and 2 had extremely low occurrence rate, suggesting that they are most likely outliers). Notably, the motion levels in dRSFC patterns 3,4 , and 5 in this low-motion dataset were comparable (Supplementary Fig. S4c), indicating that characteristic dRSFC patterns revealed in the present study were not attributed to movement.

Another limitation is that the structural connectivity was derived from the mouse brain atlas. Although highly similar, the brain structure and regional axonal projection patterns can still be different between rats and mice. However, we used ROIs at relatively large spatial scales, which generally preserve similar functions between rats and mice. In addition, since our goal is to examine whole-brain level network changes at graded anesthetic depths, subtle difference at in- dividual substructures should have limited impact on our results. It also has to be noted that the findings of the present study can only be applied to isoflurane. To generalize these findings, it is necessary to conduct a similar study using different anesthetic agents.

\section{Acknowledgments}

The authors would like to thank Dr. Pablo Perez for his technical support, and Ms. Lilith Antinori for editing the article. Dr. N.Z. is supported by R01MH098003 from the National Institute of Mental Health and R01NS085200 from the National Institute of Neurological Disorders and Stroke.

\section{Author Disclosure Statement}

No competing financial interests exist.

\section{References}

Allen EA, Damaraju E, Plis SM, Erhardt EB, Eichele T, Calhoun VD. 2014. Tracking whole-brain connectivity dynamics in the resting state. Cereb Cortex 24:663-676.

Anand A, Li Y, Wang Y, Wu J, Gao S, Bukhari L, et al. 2005. Activity and connectivity of brain mood regulating circuit in depression: a functional magnetic resonance study. Biol Psychiatry 57:1079-1088.

Barttfeld P, Uhrig L, Sitt JD, Sigman M, Jarraya B, Dehaene S. 2015. Signature of consciousness in the dynamics of restingstate brain activity. Proc Natl Acad Sci U S A 112:887-892.

Birn RM, Smith MA, Jones TB, Bandettini PA. 2008. The respiration response function: the temporal dynamics of fMRI signal fluctuations related to changes in respiration. Neuroimage 40:644-654.

Biswal B, Yetkin FZ, Haughton VM, Hyde JS. 1995. Functional connectivity in the motor cortex of resting human brain using echo-planar MRI. Magn Reson Med 34:537-541.

Boveroux P, Vanhaudenhuyse A, Bruno MA, Noirhomme Q, Lauwick S, Luxen A, et al. 2010. Breakdown of withinand between-network resting state functional magnetic resonance imaging connectivity during propofol-induced loss of consciousness. Anesthesiology 113:1038-1053.

Breshears JD, Roland JL, Sharma M, Gaona CM, Freudenburg ZV, Tempelhoff R, et al. 2010. Stable and dynamic cortical electrophysiology of induction and emergence with propofol anesthesia. Proc Natl Acad Sci U S A 107:21170-21175.

Carter AR, Shulman GL, Corbetta M. 2012. Why use a connectivity-based approach to study stroke and recovery of function? Neuroimage 62:2271-2280.

Chang C, Cunningham JP, Glover GH. 2009. Influence of heart rate on the BOLD signal: the cardiac response function. Neuroimage 44:857-869.

Chang C, Glover GH. 2009. Effects of model-based physiological noise correction on default mode network anticorrelations and correlations. Neuroimage 47:1448-1459.

Chang C, Glover GH. 2010. Time-frequency dynamics of resting-state brain connectivity measured with fMRI. Neuroimage 50:81-98.

Finn ES, Shen X, Scheinost D, Rosenberg MD, Huang J, Chun MM, et al. 2015. Functional connectome fingerprinting: identifying individuals using patterns of brain connectivity. Nat Neurosci 18:1664-1671.

Fox MD, Raichle ME. 2007. Spontaneous fluctuations in brain activity observed with functional magnetic resonance imaging. Nat Rev Neurosci 8:700-711. 
Franks NP. 2008. General anaesthesia: from molecular targets to neuronal pathways of sleep and arousal. Nat Rev Neurosci 9:370-386.

Greicius MD, Flores BH, Menon V, Glover GH, Solvason HB, Kenna H, et al. 2007. Resting-state functional connectivity in major depression: abnormally increased contributions from subgenual cingulate cortex and thalamus. Biol Psychiatry $62,429-437$.

Greicius MD, Srivastava G, Reiss AL, Menon V. 2004. Defaultmode network activity distinguishes Alzheimer's disease from healthy aging: evidence from functional MRI. Proc Natl Acad Sci U S A 101:4637-4642.

Hamilton C, Ma Y, Zhang N. 2016. Global Reduction of Information Exchange during Anesthetic-Induced Unconsciousness. [Epub ahead of print], doi: http://dx.doi.org/10.1101/074658.

Hudetz AG, Liu X, Pillay S. 2015. Dynamic repertoire of intrinsic brain states is reduced in propofol-induced unconsciousness. Brain Connect 5:10-22.

Hudetz AG, Vizuete JA, Pillay S. 2011. Differential effects of isoflurane on high-frequency and low-frequency gamma oscillations in the cerebral cortex and hippocampus in freely moving rats. Anesthesiology 114:588-595.

Hunter JV, Wilde EA, Tong KA, Holshouser BA. 2012. Emerging imaging tools for use with traumatic brain injury research. J Neurotrauma 29:654-671.

Hutchison RM, Womelsdorf T, Allen EA, Bandettini PA, Calhoun VD, Corbetta M, et al. 2013a. Dynamic functional connectivity: promise, issues, and interpretations. Neuroimage 80:360-378.

Hutchison RM, Womelsdorf T, Gati JS, Everling S, Menon RS. 2013b. Resting-state networks show dynamic functional connectivity in awake humans and anesthetized macaques. Hum Brain Mapp 34:2154-2177.

Jones DT, Vemuri P, Murphy MC, Gunter JL, Senjem ML, Machulda MM, et al. 2012. Non-stationarity in the "resting brain's" modular architecture. PLoS One 7:e39731.

Kalthoff D, Seehafer JU, Po C, Wiedermann D, Hoehn M. 2011. Functional connectivity in the rat at $11.7 \mathrm{~T}$ : impact of physiological noise in resting state fMRI. Neuroimage 54:28282839.

Keilholz SD. 2014. The neural basis of time-varying resting-state functional connectivity. Brain Connect 4:769-779.

Keilholz SD, Magnuson ME, Pan WJ, Willis M, Thompson GJ. 2013. Dynamic properties of functional connectivity in the rodent. Brain Connect 3:31-40.

Kennedy DP, Redcay E, Courchesne E. 2006. Failing to deactivate: resting functional abnormalities in autism. Proc Natl Acad Sci U S A 103:8275-8280.

Laureys S, Perrin F, Bredart S. 2007. Self-consciousness in noncommunicative patients. Conscious Cogn 16:722-741; discussion 742-745.

Lee U, Ku S, Noh G, Baek S, Choi B, Mashour GA. 2013. Disruption of frontal-parietal communication by ketamine, propofol, and sevoflurane. Anesthesiology 118:1264-1275.

Liang Z, King J, Zhang N. 2011. Uncovering intrinsic connectional architecture of functional networks in awake rat brain. J Neurosci 31:3776-3783.

Liang Z, King J, Zhang N. 2012a. Anticorrelated resting-state functional connectivity in awake rat brain. Neuroimage 59:1190-1199.

Liang Z, King J, Zhang N. 2012b. Intrinsic organization of the anesthetized brain. J Neurosci 32:10183-10191.

Liang Z, King J, Zhang N. 2014. Neuroplasticity to a singleepisode traumatic stress revealed by resting-state fMRI in awake rats. Neuroimage 103:485-491.
Liang Z, Li T, King J, Zhang N. 2013. Mapping thalamocortical networks in rat brain using resting-state functional connectivity. Neuroimage 83:237-244.

Liang Z, Liu X, Zhang N. 2015a. Dynamic resting state functional connectivity in awake and anesthetized rodents. Neuroimage 104:89-99.

Liang Z, Watson GD, Alloway KD, Lee G, Neuberger T, Zhang N. 2015b. Mapping the functional network of medial prefrontal cortex by combining optogenetics and fMRI in awake rats. Neuroimage 117:114-123.

Liu X, Duyn JH. 2013. Time-varying functional network information extracted from brief instances of spontaneous brain activity. Proc Natl Acad Sci U S A 110:4392-4397.

Liu X, Yanagawa T, Leopold DA, Fujii N, Duyn JH. 2015. Robust long-range coordination of spontaneous neural activity in waking, sleep and anesthesia. Cereb Cortex 25:29292938.

Liu X, Zhu XH, Zhang Y, Chen W. 2011. Neural origin of spontaneous hemodynamic fluctuations in rats under burstsuppression anesthesia condition. Cereb Cortex 21:374-384.

Lowe MJ, Phillips MD, Lurito JT, Mattson D, Dzemidzic M, Mathews VP. 2002. Multiple sclerosis: low-frequency temporal blood oxygen level-dependent fluctuations indicate reduced functional connectivity initial results. Radiology 224: 184-192.

Lu H, Zuo Y, Gu H, Waltz JA, Zhan W, Scholl CA, et al. 2007. Synchronized delta oscillations correlate with the restingstate functional MRI signal. Proc Natl Acad Sci U S A 104: 18265-18269.

Lustig C, Snyder AZ, Bhakta M, O’Brien KC, McAvoy M, Raichle ME, et al. 2003. Functional deactivations: change with age and dementia of the Alzheimer type. Proc Natl Acad Sci U S A 100:14504-14509.

Ma Z, Perez P, Ma Z, Liu Y, Hamilton C, Liang Z, Zhang N. 2016. Functional atlas of the awake rat brain: a neuroimaging study of rat brain specialization and integration. Neuroimage [Epub ahead of print], doi: 10.1016/j.neuroimage.2016.07.007.

MacIver MB, Bland BH. 2014. Chaos analysis of EEG during isoflurane-induced loss of righting in rats. Front Syst Neurosci 8:203.

Majeed W, Magnuson M, Hasenkamp W, Schwarb H, Schumacher EH, Barsalou L, Keilholz SD. 2011. Spatiotemporal dynamics of low frequency BOLD fluctuations in rats and humans. Neuroimage 54:1140-1150.

Mashour GA, Alkire MT. 2013. Evolution of consciousness: phylogeny, ontogeny, and emergence from general anesthesia. Proc Natl Acad Sci U S A 110 Suppl 2:10357-10364.

Mayer AR, Mannell MV, Ling J, Gasparovic C, Yeo RA. 2011. Functional connectivity in mild traumatic brain injury. Hum Brain Mapp 32:1825-1835.

Oh SW, Harris JA, Ng L, Winslow B, Cain N, Mihalas S, et al. 2014. A mesoscale connectome of the mouse brain. Nature 508:207-214.

Peltier SJ, Kerssens C, Hamann SB, Sebel PS, Byas-Smith M, Hu X. 2005. Functional connectivity changes with concentration of sevoflurane anesthesia. Neuroreport 16:285-288.

Power JD, Schlaggar BL, Petersen SE. 2015. Recent progress and outstanding issues in motion correction in resting state fMRI. Neuroimage 105:536-551.

Shirer WR, Ryali S, Rykhlevskaia E, Menon V, Greicius MD. 2012. Decoding subject-driven cognitive states with wholebrain connectivity patterns. Cereb Cortex 22:158-165.

Swanson LW. 2004. Brain Maps: Structure of the Rat Brain. New York, NY: Elsevier. 
Thompson GJ, Merritt MD, Pan WJ, Magnuson ME, Grooms JK, Jaeger D, Keilholz SD. 2013. Neural correlates of time-varying functional connectivity in the rat. Neuroimage 83:826-836.

Thompson GJ, Pan WJ, Magnuson ME, Jaeger D, Keilholz SD. 2014. Quasi-periodic patterns (QPP): large-scale dynamics in resting state fMRI that correlate with local infraslow electrical activity. Neuroimage 84:1018-1031.

Tian L, Jiang T, Wang Y, Zang Y, He Y, Liang M, et al. 2006. Altered resting-state functional connectivity patterns of anterior cingulate cortex in adolescents with attention deficit hyperactivity disorder. Neurosci Lett 400:39-43.

Tononi G. 2004. An information integration theory of consciousness. BMC Neurosci 5:42.

van Meer MP, Otte WM, van der Marel K, Nijboer $\mathrm{CH}$, Kavelaars A, van der Sprenkel JW, et al. 2012. Extent of bilateral neuronal network reorganization and functional recovery in relation to stroke severity. J Neurosci 32:4495-4507.

Vincent JL, Patel GH, Fox MD, Snyder AZ, Baker JT, Van Essen DC, et al. 2007. Intrinsic functional architecture in the anaesthetized monkey brain. Nature 447:83-86.

White NS, Alkire MT. 2003. Impaired thalamocortical connectivity in humans during general-anesthetic-induced unconsciousness. Neuroimage 19:402-411.
Whitfield-Gabrieli S, Thermenos HW, Milanovic S, Tsuang MT Faraone SV, McCarley RW, et al. 2009. Hyperactivity and hyperconnectivity of the default network in schizophrenia and in first-degree relatives of persons with schizophrenia. Proc Natl Acad Sci U S A 106:1279-1284.

Yuan BK, Wang J, Zang YF, Liu DQ. 2014. Amplitude differences in high-frequency fMRI signals between eyes open and eyes closed resting states. Front Hum Neurosci 8:503.

Zhang N, Rane P, Huang W, Liang Z, Kennedy D, Frazier JA, King J. 2010. Mapping resting-state brain networks in conscious animals. J Neurosci Methods 189:186-196.

Address correspondence to: Nanyin Zhang Department of Biomedical Engineering Pennsylvania State University W-341 Millennium Science Complex University Park, PA 16802

E-mail:nuz2@psu.edu 